\title{
PAOLO MANTEGAZZA E LA FACOLTÀ DI MEDICINA: UNA PRESIDENZA DI CAMBIAMENTO
}

\author{
GUIDO COGGI (*)
}

SunTo. - La Presidenza di Paolo Mantegazza coincise con un periodo di grande turbolenza sociale e di spinte al cambiamento e fu caratterizzata da una leadership gentile, paziente e tuttavia tenace, che rispecchiava appieno il temperamento dell'uomo. Conscio della necessità di risolvere il problema dell'affollamento studentesco e intuendo le difficoltà che esso avrebbe potuto creare negli anni della formazione clinica dei futuri medici, egli sviluppò, primo in Italia, un processo di decentramento della Facoltà in diversi Ospedali pubblici e privati, che venne poi seguito anche da altre Facoltà e che rappresentò un significativo cambiamento rispetto alla tradizione accademica italiana. L'aspetto più innovativo e complesso della politica dei Poli Universitari fu rappresentato dalla necessità di contemperare le esigenze didattico-scientifiche dell'Università con quelle Ospedaliere, tradizionalmente più volte alla soluzione pratica dei problemi di salute dei pazienti che non alla ricerca o all'insegnamento. Di fatto, questa necessità divenne, sotto la guida di Mantegazza, una grande opportunità sia per il mondo accademico che per quello ospedaliero: ne derivò infatti un mutuo scambio di esperienze sia didattiche sia assistenziali sia, col tempo, scientifiche, che certamente giovarono ad entrambi i protagonisti del processo di integrazione.

$$
* * *
$$

ABSTRACT. - The years during which Paolo Mantegazza served as Dean of the Medical Faculty, the Country was involved in a considerably large social turmoil, under the pressure of the so called student revolution, which was spreading through Europe. Such a movement, albeit very often out of control (with dangerous deviations), still was, in its basic and best aspects, the expression of an urgent need for innovation and social change. Mantegazza, who was a very qualified scientist in Pharmacology, perceived by intuition the need for a controlled change in the style and action of his office, and used a "gentle leadership" to carry on consistent innovations in the traditional "academic" policy of the Faculty. His major achievement was twofold: first, he was able to expand the Faculty into city hospitals other than the traditional Main Central University Hospital, thus allowing students to perform clinical practice in a new environment, more patient oriented than the classical one; second, this expansion offered teachers

(*) Università degli Studi di Milano, Italia. E-mail: guido.coggi@alice.it 
and researchers the opportunity to practice a less formal teaching, paying more attention, both from a clinical, scientific and teaching point of view, to the complexity of the patient and his/her social and personal context, with relevant consequences on the educational approach and to teacher-students relationships.

Una delle caratteristiche più rilevanti della personalità di Paolo Mantegazza, che influenzarono fortemente la sua azione di Preside e poi di Rettore, fu la capacità di leggere e interpretare i cambiamenti propri della sua epoca adattandoli alle esigenze del suo ufficio, cogliendone e valorizzandone gli aspetti più positivi $\mathrm{o}$, comunque, meno negativi.

La sua presidenza della Facoltà di Medicina, iniziata nel 1975, rappresentò il giro di boa nei tradizionali rapporti tra Società civile e Facoltà medica e di conseguenza tra la prima e l'Università: con Mantegazza, l'Accademia si apriva alla realtà sociale e ai suoi cambiamenti.

Erano quelli gli anni di fuoco che stavano letteralmente scardinando la nostra società e il nostro Paese. Si pensi agli attentati, ai sequestri, alle stragi dell'Italicus, di Bologna, di Brescia, al sequestro del Presidente Moro e a tutti gli altri drammatici episodi della cronaca di quei tempi.

Furono anni che incisero profondamente nella percezione dei nostri valori: anni che, nella loro drammatica tragicità, testimoniarono un disagio profondo specie nei giovani e dettero inizio a un ripensamento globale dei principi su cui ci eravamo basati, a una riflessione critica sul futuro della nostra società.

Su questo sfondo comparve Mantegazza, un uomo di semplici ma genuine origini, che non ha mai rinnegato, anzi essendone fiero, e con una brillane carriera scientifica alle spalle.

Non saprei dire quali elementi portarono Mantegazza dai laboratori di ricerca alla Presidenza della Facoltà. Certamente, un peso non trascurabile lo ebbe l'Istituto di Farmacologia, potente struttura accademica e scientifica creata dal Prof. Trabucchi: ma non sottovaluterei la popolarità di cui godeva quel farmacologo dagli occhi miti, che gli derivava dalla sua frequentazione con numerosissimi giovani clinici che proprio in quell'Istituto maturavano un'esperienza scientifica. Si aggiunga a ciò la fitta rete di rapporti anche personali, amicali, con esponenti del mondo extrauniversitario, soprattutto d'ispirazione cattolica.

La frequentazione con il mondo clinico contribuì, oltre che alla sua elezione, alla sua sensibilità verso la medicina clinica. Non si spiegherebbe altrimenti come questo scienziato potesse, una volta eletto, mostrare 
un'attenzione ai problemi della medicina quanto Paolo Mantegazza. E ciò ebbe importantissimi effetti sull'evoluzione della Facoltà.

La sua naturale semplicità, che peraltro nascondeva una forza, una caparbietà, una pazienza non comuni, gli consentì di avviare un processo che chiamerei di "demitizzazione istituzionale", nel senso di introdurre una "leardeship gentile" ed eticamente molto forte, meno formale, più personale e umana.

Non va sottostimato questo dettaglio: il suo approccio così apparentemente semplice e quasi "familiare", da buon padre, fu lo strumento con cui egli affrontò gli anni di piombo dando alla Facoltà e alla Presidenza uno stile del tutto nuovo.

Il suo grande merito, in quel contesto di furibonda violenza, è stato quello di aver resistito agli eccessi ma di non averli mai affrontati con atteggiamenti conformisti o autoritari: credo che l'esperienza di padre di numerosi figli in età scolastica e la frequentazione dei loro amici e compagni gli permettesse di capire o intuire l'evoluzione della società e della gioventù.

E' come se egli avesse capito che anche l'Accademia aveva bisogno di una difesa, una protezione "paterna" da quella violenza, anche e soprattutto perché questa colpiva soprattutto i giovani, gli studenti, e andava dunque capita anche se frenata e talora anche contrastata.

Dunque, l'individuo, la sua storia, la sua sensibilità, le sue esperienze familiari anche dolorose, e il contesto sociale, complesso e turbolento: queste le cifre, io penso, che caratterizzarono la presidenza della Facoltà di Mantegazza.

Il segnale del cambiamento si ebbe già nelle primissime sedute di Facoltà. Si dibatteva il tema della permanenza dei professori fuori ruolo alla direzione degli istituti policattedra. Tra gli Istituti interessati vi era proprio quello di Farmacologia, fino allora diretto dal Prof. Trabucchi. E fu eletto Direttore Mantegazza, contro ogni tradizione: segnale di un'evoluzione all'interno dei rapporti gerarchici e di Scuola, e nello stesso tempo origine dell'affetto e del rispetto che da allora in poi la Facoltà, specialmente nei suoi più giovani componenti, portò al nuovo Preside.

Il vero cambiamento, tuttavia, si ebbe con lo sviluppo dei Poli, cioè con il coinvolgimento di Ospedali e personale non Universitari nella formazione del medico e con l'inserimento, in questi stessi Ospedali, di personale docente universitario. Si attribuisce questo passo al Rettorato di Mantegazza, ma in realtà esso fu l'elemento di cambiamento più rilevante della sua Presidenza. 
A dire il vero, l'espansione della Facoltà negli Ospedali cittadini era già stata avviata da circa due anni prima, ad opera del preside Ratti.

Le ragioni di questa espansione furono, all'inizio, meramente logistiche, da ricercarsi nell'aumento degli iscritti alla Facoltà: si pensi che in quegli anni, a seguito della liberalizzazione degli accessi, gli studenti di Medicina erano 17.000, con una media di 2-3000 accessi l'anno. Da qui, la ricerca di spazi almeno per l'insegnamento teorico: basti pensare che le lezioni dei primi anni, quelle di anatomia, di chimica, di fisiologia, si tennero anche in sale cinematografiche.

Mantegazza intuì che il problema dell'affollamento studentesco era ben più complesso e avrebbe avuto effetti tragici sulla formazione dei futuri medici negli anni cosiddetti clinici, quelli del contatto con il malato: la tradizionale sede clinica della Facoltà, costituita dall'Ospedale Policlinico e dagli Istituti Clinici di Perfezionamento, ora si rivelava drammaticamente insufficiente e pericolosamente incapace di offrire una reale esperienza clinica.

Fu così che con Mantegazza si avviò una stagione del tutto nuova, per la prima volta in Italia, dei rapporti tra Facoltà e mondo della Sanità pubblica e privata: l'integrazione tra mondo ospedaliero e quello universitario. Un obiettivo molto difficile per le differenze profonde tra i due mondi.

Infatti, mentre nella sede clinica tradizionale l'Università, di fatto, condizionava, con le proprie esigenze di ricerca e d'insegnamento, tutta l'organizzazione del processo assistenziale, negli altri Ospedali, cui Mantegazza si accingeva a chiedere accoglienza per studenti e docenti, le regole di gestione non si erano formate sul modello universitario, ma su un modello del tutto indipendente che poco o nulla aveva a che vedere con la formazione dei futuri medici.

Questa fu la sfida più critica e direi storica: da una parte le esigenze Universitarie, nate e sviluppatesi in linea con la missione prevalentemente scientifica e didattica; dall'altra, la natura propria dell'Ospedale, che si limitava alla cura dei malati, escludendo, di fatto, sia la formazione sia la ricerca.

Da qui anche la prospettiva che le regole dei due sistemi potessero confliggere, come spesso conflissero, e la preoccupazione che l'intero progetto potesse fallire per motivi i più diversi, culturali, sindacali, gestionali. Si trattava di un progetto ad altissimo rischio, che richiedeva una specialissima sensibilità e abilità diplomatica e politica.

Mantegazza comprese subito la complessità del compito. 
Lo compresero i più tradizionalisti della Facoltà, docenti di tutto rispetto, che vedevano nella possibile coesistenza fra tradizione accademica e tradizione puramente ospedaliera, il rischio, anzi la certezza di un inquinamento e "snaturamento" della natura universitaria, della perdita di autonomia o addirittura, come disse un anziano professore in una seduta di Facoltà, di "umiliazione".

Reazioni, queste, che danno la dimensione del trauma, dello shock, dell'incertezza di fronte ad una situazione cui non era possibile porre rimedio in alcun altro modo.

Qui giocò il pragmatismo, il senso del necessario, del tangibilmente necessario propri di Mantegazza: il suo persuasivo understatement gli consentì di resistere alle critiche e alle paure.

Fu veramente un fatto straordinario che un uomo di scienza potesse avere la sensibilità politica e il coraggio di gestire, per la parte accademica, il profondo cambiamento che i tempi esigevano: le decisioni politiche avevano creato l'affollamento della Facoltà, ma toccava all'Università -in questo caso a Mantegazza- il compito di risolvere, nell'interesse degli studenti, i problemi che quelle decisioni avevano prodotto.

Mantegazza utilizzò la propria autorevolezza, pazienza e credibilità per consentire un'integrazione tra mondo universitario e mondo ospedaliero. La negoziazione con gli enti ospedalieri sui criteri cui doveva ispirarsi la convivenza tra due mondi spesso in contrasto tra loro, fu il suo principale impegno, conscio com'era della irrinunciabilità del cambiamento.

I problemi che questa storica svolta, poi seguita anche in altre sedi Italiane, comportò furono, infatti, numerosi: e, inaspettatamente, la loro soluzione non solo riuscì a garantire agli studenti una formazione clinica, ma produsse anche cambiamenti nella docenza universitaria, che si rese più coerente con l'evoluzione della sanità che nel frattempo si stava attuando.

Per i docenti, in gran parte giovani, l'esperienza si rivelò, nonostante le iniziali difficoltà, estremamente costruttiva; si entrava tuttavia in una realtà del tutto nuova: terrae incognitae.

La convivenza con i colleghi ospedalieri si presentò all'inizio difficile: essi vedevano negli universitari dei "colonizzatori" e i più giovani temevano un ostacolo alle loro naturali aspirazioni di carriera.

La differenza con la sede storica del Policlinico era molto evidente: qui l'Università era dominante, forse fin troppo, e comunque l'approccio era fondamentalmente scientifico e didattico. 
Negli Ospedali la situazione era completamente diversa: le amministrazioni ospedaliere, tutte di estrazione politica, non potevano usare due pesi e due misure per gli universitari e per gli ospedalieri, così che gli universitari si dovettero adattare a una disciplina totalmente nuova e al rispetto di norme per noi inusuali: per citare un solo piccolo esempio, il timbro del cartellino orario che, obbligatorio per il personale ospedaliero, non poteva non essere applicato anche al personale universitario. Si trattò di un punto caldissimo, che causò infinite polemiche. Oppure il calcolo delle ore di assistenza verso quello delle ore d'insegnamento e di ricerca. $\mathrm{O}$ ancora le assenze per partecipare alle adunanze di Facoltà. O la turnazione nelle guardie mediche, che contrastava spesso con le esigenze didattiche e di ricerca.

Al Policlinico l'attenzione al malato era certamente presente, ma subordinata alla ricerca e alla didattica.

Questo non accadeva in un Ospedale, in cui l'ammalato era solo oggetto di diagnosi e cura, non di studio, di ricerca o d'insegnamento: nonostante ciò, questo approccio prevalentemente assistenziale a poco a poco riuscì a coniugarsi con quello didattico-scientifico. Si arrivò, progressivamente, a una nuova concezione del malato da entrambe le parti: da parte universitaria una maggiore attenzione agli aspetti personali del malato e alle sue primarie esigenze di cura: dall'altra, una nuova percezione del paziente come motivo di riflessione, di studio, di ricerca.

Nasceva così un nuovo modo di essere universitari medici: più vicini alla realtà, alle tematiche organizzative e gestionali degli ospedali. Il contatto con malati non selezionati, di ogni genere, anche non interessante dal punto di vista scientifico, chiamava a una nuova attenzione e sensibilità.

A questi aspetti in gran parte positivi, peraltro, continuavano ad aggiungersi continui problemi da risolvere, riguardanti gli organici, la ricerca di strutture per la didattica, di aule, di segreterie, nonché la necessità di chiedere la collaborazione dei medici ospedalieri nella didattica pratica, con la conseguente complessità di rapporti interpersonali che ne derivavano.

Negli anni della Presidenza Mantegazza, e poi nei successi fino al 2006, non ci fu mai un Ordine del Giorno delle adunanze di Facoltà che non recasse il punto: "Rapporti convenzionali con enti ospedalieri".

E questa voce, si badi bene, apparentemente di ordinaria amministrazione, nascondeva in realtà un'intricatissima rete d'incontri, scontri, riunioni in assessorato o in presidenza, di spinte e controspinte politiche, di compromessi di cui Mantegazza era il paziente tessitore. 
Alla fine la sua politica vinse: le resistenze, sia interne sia esterne, vennero superate, $i$ rapporti con i medici ospedalieri divennero amichevoli e collaborativi, e a poco a poco l'influenza dell'Università, di una nuova Università, si andò affermando. La qualità dell'assistenza migliorò, lo studio dei malati divenne più riflessivo, la ricerca cominciò ad attrarre anche i giovani medici ospedalieri, gli spazi furono reperiti.

L'esperienza milanese dei Poli, voluta e condotta da Mantegazza, ebbe peraltro un altro importante effetto sul piano nazionale. Fu infatti proprio dalla nuova convivenza tra mondo ospedaliero e mondo universitario, nonché dalla spinta che proveniva dagli studenti, critici verso la tradizionale formazione accademica, che nacque una nuova sensibilità verso le esigenze di una didattica moderna, meno formale, più attenta al rapporto medico-paziente, più sensibile alle esigenze di esperienze personali da parte degli studenti.

Questo cambiamento aveva cominciato ad affermarsi in tutto il mondo: già negli anni 70 era nato il problema della ricerca di nuovi modelli di formazione in medicina. L'aumento delle conoscenze, la necessità di una sensibilità nuova, lo sviluppo delle tecnologie, la complessità stessa della persona malata, avevano favorito gli studi in questo campo, alla ricerca di metodologie di formazione nuove e più aderenti alla realtà.

Si trattava di mantenere l'impianto scientifico della formazione medica, affiancandogli una nuova attenzione ai problemi del ragionamento clinico, del management, della gestione economica della salute, della comunicazione medico-paziente, della stessa prevenzione, dell'attenzione agli stili di vita, tutte tematiche sostanzialmente ignorate dalla formazione classica. Insomma, i tempi richiedevano che alla formazione classica si affiancasse una formazione alla professionalità medica.

L'obiettivo era quello di fare un medico capace di intercettare culturalmente e soddisfare, sia scientificamente che umanamente, i bisogni di salute della popolazione. Un concetto del tutto nuovo da noi, già allora ben presente nelle migliori Università europee e anglosassoni e sostenuto, nella facoltà milanese, dal Prof. Maccacaro, che proponeva il concetto, allora del tutto innovativo e altamente provocatorio, di "Facoltà capovolta", cioè di una Facoltà che partisse dai problemi dei cittadini, sani o malati, per arrivare poi alle tematiche scientifiche, biologiche o cliniche, che quei problemi suscitavano.

Ebbene, nel secondo mandato di Mantegazza, il rinnovamento della didattica prese piede anche da noi: e ciò non sarebbe accaduto senza i Poli. Fu proprio lì che la nuova osmosi che si era verificata tra 
Università e Sanità rese possibile l'innovazione didattica, che portò la Facoltà milanese a essere la prima per tempestività, ma anche per importanza, in tutto il nostro paese in questo campo e a svolgere un ruolo significativo anche sul piano normativo e legislativo.

Intanto, accanto allo sviluppo dei Poli, si andavano moltiplicando le convenzioni con decine di Ospedali anche fuori della cerchia cittadina per consentire agli specializzandi una pratica che richiedeva di necessità una casistica molto ampia. E anche qui, Mantegazza dovette adoprarsi per mantenere equilibrio tra interessi territoriali o locali, cui la politica non era sempre estranea, che minacciavano di porre sempre sotto ricatto l'Università, con il rischio di annullare la convenzione. Rischio poco probabile, visti gli interessi e i vantaggi che derivavano anche alla classe ospedaliera, ma certamente da non sottovalutare.

Non è possibile elencare tutte le iniziative che si realizzarono durante quella Presidenza: citerò solo la nascita del nuovo corso di Laurea in odontoiatria, prima laurea sanitaria a indirizzo professionalizzante, che permetteva agli studenti di curare materialmente e personalmente i pazienti e che si poneva nella stessa linea di tendenza della didattica innovativa in Medicina e Chirurgia.

Né si può dimenticare che, nel successivo Rettorato, l'esperienza dei Poli consentì, sempre sotto la guida di Mantegazza, la nascita di nuove Facoltà: Brescia, Milano Bicocca, Vita e Salute-S. Raffaele e, in tempi successivi e con modalità diverse, la Humanitas University.

Insomma, una presidenza densa, ricca, concreta e incisiva, innovatrice.

Mantegazza non era un uomo semplice, tutt'altro: egli aveva sempre la misura del possibile. La sua conoscenza del mondo clinico, la sua vicinanza ai giovani, figlioli e amici dei figlioli, la sua ispirazione profondamente religiosa, caritatevole, ma pragmaticamente ancorata all'oggi, gli facevano comprendere il cambiamento dei tempi e lo portavano ad accettarlo e a farlo accettare ai più tradizionalisti.

I cambiamenti prodotti dalla Presidenza di Paolo Mantegazza s'inserirono a pieno titolo nel tema della complessità, così forte e presente nella società moderna, ma ancor più nella realtà della malattia $\mathrm{e}$ della persona malata.

Mantegazza, da scienziato e da medico, seppe muoversi in questa complessità, agendo a favore di un mondo, quello dei giovani, che gli fu sommamente caro e sul quale aleggiò continuamente l'amore per i suoi figli. 